
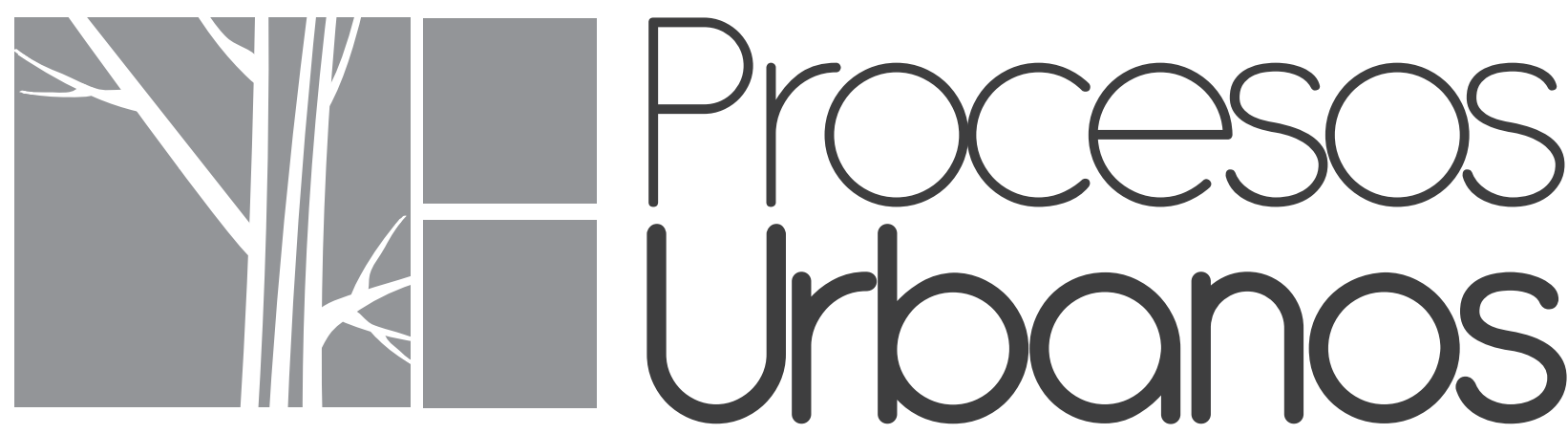

Revista de Divulgación Científica 


\section{Revista Procesos Urbanos}

Facultad de Ciencias Básicas, Ingeniería y Arquitectura.

ISSN: 2422-085X

Sincelejo, Enero - Diciembre de 2014

Piedad Martínez Carazo

Rectora

\section{Lidia Flórez de Albis}

Vicerrectora Académica

\section{Jhon Víctor Vidal}

Director de Investigaciones

\section{Pedro Arturo Martínez Osorio}

Editor

\section{Comité Editorial:}

PhD. Alexander Niño Soto. Universidad Antonio Nariño

PhD. Jorge Ramírez Nieto. Universidad Nacional de Colombia

Msc. Juan Carlos Pergolis. Universidad Católica de Colombia

PhD. Piedad Martínez Carazo. Corporación Universitaria del Caribe, CECAR

PhD. Emiro F. Martínez. Universidad de York, Canadá

\section{Comité Científico:}

Msc. Gloria Aponte García. UPB, Medellín

Msc. Jorge Vásquez Muñoz. UPB, Medellín

PhD. Jorge Gómez Ricardo, Universidad del Magdalena

\section{Editorial CECAR}

\section{Libia Narváez Barbosa}

Coordinadora Editorial

\section{Eduardo Támara Galván}

Corrector de Estilo

\section{Alejandrina Jaramillo y Roger Goez Castillo}

Diseño Gráfico

Diagramación e Impresión:GRÁFICAS DEL CARIBE S.A.S.

Cra. 1B No. 40-42 Montería Tel. (57) (4) 7826622 Telefax (57) (4) 7817112

Email: diseno@graficaribe.co

\section{Dirección:}

Carretera Troncal de Occidente

Kilómetro 1 Vía a Corozal

Tel: 280402928040172804018 Ext 1115

www.cecar.edu.co

http://cecar.edu.co/revista-procesos-urbanos 


\title{
POLÍTICAS E INSTRUMENTOS NORMATIVOS. ESTRATEGIAS AMBIENTALES ENFOCADAS HACIA UN ORDENAMIENTO BASADO EN LA PARTICIPACIÓN
}

\author{
Policies and regulatory instruments. An environmental strategies focused on \\ management based in participation
}

Héctor Enrique Guzmán Luján ${ }^{1}$

Fecha: Recibido febrero 28 de 2014 / Aceptado abril 29 de 2014

\section{RESUMEN}

Día a día la opinión pública contempla cómo Colombia, país mega diverso y dotado de gran riqueza en biodiversidad, va disminuyendo cualitativa y cuantitativamente sus recursos, afectando no solamente la calidad de vida en nuestras principales ciudades, sino la posibilidad de utilizar esa riqueza racionalmente como motor de desarrollo. Este artículo plantea como objetivo, una reflexión sobre la necesidad y pertinencia de replantear las estrategias ambientales utilizadas en la actualidad, y pregunta si realmente éstas impiden el paulatino deterioro de los recursos naturales, y si efectivamente garantizan el derecho constitucional del artículo 79, denominado derecho a gozar de un medio ambiente sano. A nivel metodológico, este trabajo surge de una revisión documental, y plantea como discusión la reflexión sobre estrategias ambientales en el ámbito nacional e internacional para afrontar la problemática ambiental en el sector productivo. Como conclusión destaca que en Colombia, a pesar de contar con múltiples instrumentos coercitivos en materia ambiental, éstas no se constituyen en una solución de fondo a las problemáticas que atacan, es necesaria una política en la materia, que pueda crear herramientas de mercado, acuerdos de autogestión y así fortalecer las relaciones entre lo público y privado.

Palabras clave: Estrategias Ambientales, Biodiversidad, Producción y Consumo Sostenible.

\begin{abstract}
Day after day the public opinion contemplates how Colombia, diverse country and gifted of great wealth in biodiversity, is diminishing qualitatively and quantitatively his resources, affecting not only the quality of life in our principal cities, but the possibility of using this wealth rationally as engine of development. This article raises as aim, a reflection on the need and relevancy of restating the environmental strategies used at present and asks, if really these prevent the gradual deterioration of the natural resources, and if really they guarantee the constitutional law of the article 79, named right to enjoy a healthy environment. To methodological level, this work arises from a documentary review, and raises as discussion the reflection on environmental strategies in the national and international area to confront the environmental problematics in the productive sector. As conclusion emphasizes that in Colombia, in spite of possessing multiple coercive instruments in environmental matter, these are not constituted in a solution of bottom to the problematic ones that they attack, a politics is necessary in the matter, which could create tools of market, agreements of automanagement and this way strengthen the relations between the public and private thing.
\end{abstract}

Keywords: $\begin{aligned} & \text { Environmental strategies, } \\ & \text { biodiversity, production and sustainable } \\ & \text { consumption. }\end{aligned}$

${ }^{1}$ Abogado Magíster en Gestión Política - Medioambiental, Especialista en Derecho Ambiental 
PROCESOS URBANOS - Revista de Divulgación Científica Vol. 1 Enero - Diciembre 2014 (63 - 70)

\section{INTRODUCCIÓN}

La problemática asociada a la degradación ambiental de nuestro entorno, no es una premisa futuro, es un hecho inocultable que afecta el suministro de recursos naturales y por ende la misma supervivencia del hombre; no pretendemos ser apocalípticos, pero si no se toman medidas concretas para revertir o al menos paliar la situación, nos veremos avocados a unas consecuencias imprevisibles para la vida en la tierra, por el aumento de la temperatura, lo que traería como consecuencia un incremento en el número de episodios de fenómenos naturales como huracanes, inundaciones, desaparición de territorios insulares, el derretimiento de los casquetes polares, entre otros.

Nuestro país no es ajeno a esta degradación, vemos a manera de ejemplo, que gran parte de las causas de los desastres naturales que se presentan en el territorio nacional, como las inundaciones o los constantes deslizamientos de tierra, se deben en gran medida a la pérdida o degradación de ecosistemas estratégicos, a prácticas de deforestación o manejo inadecuado del uso del suelo, entre otros.

Por lo tanto, debemos replantear las estrategias ambientales utilizadas en la actualidad y preguntarnos si efectivamente impiden el paulatino deterioro de los recursos naturales y si verdaderamente garantizan el derecho constitucional del artículo 79, denominado derecho a gozar de un medio ambiente sano. Así las cosas, proponemos en este artículo algunas ideas que pueden hacerle frente a la problemática.

\section{Estrategias ambientales}

Tradicionalmente las estrategias ambientales orientadas a los diversos sectores productivos se han enfocado a la utilización de instrumentos de comando y control, entendiendo la noción como los mandatos, prohibiciones, limitaciones, condicionamientos o cargas necesarias para asegurar el orden público, la concordia y la convivencia ciudadana en materia ambiental.
No obstante, una parte de la doctrina afirma que estos instrumentos son insuficientes teniendo en cuenta que son incapaces de seguir el ritmo frenético de los avances científicos, técnicos y de las múltiples complejidades de la temática ambiental, en la que en el día a día se presentan nuevas formas de contaminación o de afectación a los recursos naturales.

Así mismo, se argumenta que el sistema público de inspección y vigilancia del cumplimiento de la normativa no puede controlar el funcionamiento de las actividades industriales debido cada vez a la mayor especialización y complejidad técnica, así como a la carencia de recursos públicos (humanos económicos, financieros), en consecuencia, la organización administrativa se ve superada por la realidad de las necesidades del control industrial.

Frente a la situación, se proponen políticas e instrumentos que se orienten y den un giro hacia un ordenamiento que privilegie la prevención, la cooperación, la participación y la aceptación de sus destinatarios por encima de la represión, la prohibición y la orden. En consecuencia, se debe avanzar hacia una estructura normativa más cauta, flexible y abierta; un derecho cuyas intervenciones asuman un enfoque más preventivo que represivo (principio de precaución) que antes que imponer o prohibir, estimule, aconseje e informe (función promocional del derecho), y que antes que definir una regulación exhaustiva y cerrada de una materia, acuda a cláusulas generales (conceptos jurídicos indeterminados) o remita a fórmulas de autorregulación ${ }^{2}$.

Igualmente se debe dar el salto de un derecho protagonizado por la administración, basado en mecanismos jerárquicos de intervención y coacción, a un derecho más flexible y eficiente caracterizado por su apertura a los diferentes agentes implicados, al uso de instrumentos de carácter voluntario y convencional, y cuya finalidad, más que imponer conductas a sus destinatarios,

\footnotetext{
${ }^{2}$ Santaella Héctor Lecturas sobre Derecho del Medio Ambiente. Universidad Externado de Colombia. 2008 
sea estimularlas en defensa de una mayor protección al entorno ${ }^{3}$.

Aunque existen diversas estrategias para afrontar la crisis ambiental, en este trabajo vamos a enfocarnos en dos de ellas, las cuales consideramos, pueden dar mejores resultados en la protección y correcta utilización de los recursos naturales, que las tradicionales basadas en la represión.

Estos son acuerdos de autorregulación o autogestión y el desarrollo del consumo sostenible, enfocado a la utilización racional de la oferta de biodiversidad.

\section{Acuerdos de autogestión}

\section{- Ámbito internacional}

En el ámbito europeo una de las primeras referencias a este tipo de iniciativas de celebración de acuerdos ambientales, la encontramos en la política ambiental alemana del año de 1975, denominada Programa federal para la gestión de residuos, el cual, se basa en tres principios: a) prevención (preferencia por la minimización antes que por la reutilización) b) cooperación (preferencia por la iniciativa privada voluntaria, antes que por la imposición normativa obligatoria) y c) contaminador pagador ${ }^{4}$.

La política medioambiental de la Comunidad Europea, se ha basado fundamentalmente en los programas de acción, programas que datan desde 1973, sin embargo para el caso que nos ocupa (auto regulación) debemos mencionar el Quinto Programa Comunitario en materia de medio ambiente establecido para el periodo de 1993 - 2000.
La importancia de este programa radicó en que se alejó de las regulaciones e introdujo el concepto de responsabilidad compartida entre las administraciones y las empresas, las cuales, presionadas por las exigencias de la sociedad, se auto regulan a través de eco instrumentos voluntarios lo que ha mejorado su gestión medioambiental.

En el apartado 19 del resumen del programa se establece que "Mientras que las medidas sobre medio ambiente que se han venido adoptando hasta ahora, tendían a ser de carácter prohibitivo e insistían en impedir una determinada actuación, la nueva estrategia se inclina más por fomentar una actuación de colaboración, planteamiento que refleja la concienciación cada vez mayor de la industria y el mundo de los negocios de que por un lado, son en gran medida responsables de los problemas del medio ambiente, pero por otro lado, pueden y deben contribuir a solucionarlos. El nuevo planteamiento se va a traducir, en particular en un diálogo más estrecho con la industria y en la promoción, en las circunstancias adecuadas, de acuerdos voluntarios u otras formas de auto regulación".

\section{- Ámbito nacional}

En el ámbito colombiano, nuestra Constitución Política adopta el principio del Desarrollo Humano Sostenible, con el fin de conciliar las necesidades de mejorar calidad de vida de la población y el crecimiento económico. Así mismo, le asigna al Estado, al igual que a todas las personas, la obligación de proteger y conservar los recursos naturales de la Nación.

\footnotetext{
${ }^{3}$ Santaella Héctor citando a Koplfer en Lecturas sobre Derecho del Medio Ambiente. Universidad Externado de Colombia. 2008

${ }^{4}$ Betancor Rodríguez Andrés Pag 171. Instituciones de Derecho Ambiental Editorial la ley Madrid 2001. Introducido por la OCDE, en sucesivas recomendaciones adoptadas en 1972 (98), 1974 y 1989 (99), e impulsado en el ámbito comunitario a partir singularmente de la Recomendación 75/436 Euratom del 3 de marzo de 1975 y consiste en "conforme al principio de quien contamina paga, se entiende por responsable de la contaminación a la persona física o jurídica sometida a Derecho privado o público que directa o indirectamente deteriora el medio ambiente o crea las condiciones para que se produzca dicho deterioro, tratándose, básicamente, de evitar en principio que la política ambiental de protección del medio ambiente se base en subvenciones y ayudas estatales y que se atribuya a la comunidad la carga de la lucha contra la contaminación, siendo imputable al contaminador el costo de las medidas necesarias para la eliminación de la contaminación o para su reducción hasta estándares o medidas equivalentes de objetivos de calidad ambiental.
} 
Dentro de los principios fundamentales de la política ambiental, artículo 1 de la Ley 99 de 1993, se establece entre otros, que las acciones encaminadas a proteger, conservar y recuperar el medio ambiente, son tarea conjunta del Estado, el sector privado, la comunidad y las organizaciones no gubernamentales.

Mediante la creación del Sistema Nacional Ambiental (SINA), artículos 2 y 4 de la Ley 99 de 1993, se garantiza la adopción y ejecución de programas y proyectos para poner en marcha los principios ambientales contemplados en la Ley.

El artículo 5 de la Ley 99 de 1.993 consagró la posibilidad de establecer mecanismos de concertación del Ministerio de Ambiente, Vivienda y Desarrollo Territorial con el sector privado para ajustar las actividades de éste a las metas ambientales previstas por el Gobierno, y definir los casos en que haya lugar a la celebración de convenios para la ejecución de planes de cumplimiento con empresas privadas, para ajustar tecnologías y eliminar o mitigar factores contaminantes, así como fijar las reglas para el cumplimiento de los compromisos derivados de dichos convenios.

El documento CONPES 2750 de diciembre 21 de 1994 adopta como programa de acción, promover la producción limpia, previendo planes de reconversión industrial para garantizar la adopción de métodos de producción sostenibles.

Los Planes Nacionales de Desarrollo han establecidola concertación como instrumento que permita incorporar la variable ambiental en las políticas, programas y proyectos de todos los sectores del Estado y la sociedad. Igualmente, incorporan la gradualidad como una estrategia indispensable, dada la magnitud de los problemas ambientales y el carácter de largo plazo de su atención.

La política nacional de producción más limpia, adoptada por el Consejo Nacional Ambiental en agosto de 1997, tiene como objetivo prevenir y minimizar los impactos y riesgos a los seres humanos y al ambiente, para garantizar la protección ambiental y el crecimiento económico, mediante la optimización en el uso de los recursos naturales, materias primas y energía, la adopción de tecnologías limpias y las prácticas de mejoramiento continuo.

Según el Ministerio de Ambiente y Desarrollo Sostenible ${ }^{5}$ los resultados logrados después de 14 años de implementación de la política de producción más limpia pueden calificarse en términos generales como satisfactorios e igualmente puede afirmarse que el sector productivo del país viene respondiendo de manera positiva a los retos y compromisos adquiridos. El empresario ha venido comprobando que la inclusión de la variable ambiental tiene un valor agregado en su esquema de producción y ya no es considerado como una carga impositiva, observándose cada vez más un enfoque ambiental preventivo y un manejo responsable y competitivo en su desarrollo corporativo.

Un esquema interesante es el estructurado en la capital del país, para la puesta en marcha de implementación de convenios de concertación para una producción más limpia en el Distrito Capital, el cual contienen los siguientes principios.

- Integralidad

- Concertación

- Internalización de los costos ambientales

- Gradualidad

- Solidaridad

Y desarrolla una estructura mínima para la celebración de convenios de la siguiente manera:

- Justificación

- Objeto

- Actores

- Entidades de Apoyo

- Alcance

- Acuerdos y Compromisos

- Autoridad Ambiental

- Empresas

- Investigación y Desarrollo

- Criterios Operacionales

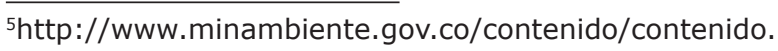


- Comité Operativo

- Terminación del Convenio

- Duración del Convenio

- Firmas

\section{Consumo Sostenible}

La política nacional de producción y consumo sostenible, expedida por el Ministerio de Ambiente Vivienda y Desarrollo Territorial en el 2010, plantea los siguientes cambios de paradigmas para la gestión ambiental en los avances internacionales de la siguiente manera:

Por medio de estos avances y muchos otros no mencionados, la producción más limpia y el consumo sostenible se han convertido en nuevos paradigmas para la gestión ambiental, lo que ha contribuido a la evolución de su enfoque. Algunos ejemplos de cambios en el paradigma de gestión ambiental que muestran los avances internacionales son: (i) Instrumentos de comando y control versus incentivos del mercado, (ii) Optimización de procesos versus optimización del ciclo de vida del producto y negocios sostenibles, (iii) Gestión de residuos versus gestión de materia prima, (iv) Empresas individuales versus cadenas productivas, redes de empresas y simbiosis industrial $y,(v)$ Cultura de consumo versus cultura del consumo sostenible.

\section{- Consumo - Crisis Ambiental}

Para comprensión del consumo y de los problemas ambientales que de este se derivan, debemos precisar que entendemos por consumo el gasto de aquellas cosas que con el uso se extinguen o se destruyen, y que necesariamente sin importar su magnitud, tiene efectos sobre el medio ambiente natural.

Desde los inicios en la historia de la humanidad, se vienen presentando impactos por el consumo en los recursos naturales, teniendo en cuenta que el hombre antiguo debía consumir para sobrevivir; no obstante, el impacto ambiental generado en este modelo de subsistencia no agotaba la base de regeneración de los mismos, es decir, existía un respeto casi religioso en los pueblos antiguos por el concepto de cadena trófica, es decir, aquella cadena en la que los consumos se superponen y engloban unos a otros, no tiene más fin que el de mantener y reproducir el ciclo donde se inscribe y produce constantemente una compleja biocenosis (conjunto de interacciones entre los seres vivos que pueblan un medio geofísico).

Posteriormente, debemos situarnos en el último tercio del siglo XVIII en el cual se presentó en los países occidentales una serie de transformaciones profundas de orden económico, social político y cultural que determinaron el tránsito del modo antiguo de sociedad a un nuevo modelo de esquema, denominado sociedad industrial, a partir de la Revolución Industrial, cuyo componente característico es el llamado industrialismo, el cual se describe como la búsqueda de una productividad siempre creciente del trabajo, mediante la innovación técnica y la organización racional de la mano de obra.

Podemos afirmar, sin temor a equivocarnos, que el industrialismo es una de las causas de la crisis ecológica generada por el consumo en la actualidad, porque cambió sustancialmente la dependencia del hombre por la naturaleza; en otras palabras, la domesticó, concepto no ajeno a la polémica, mediante la aplicación de los conocimientos científicos y técnicos y necesariamente elevó la calidad de vida de la sociedad en ese momento.

Durante el desarrollo de siglo XX y a partir de la segunda guerra mundial, la gran población comienza consumir más allá de lo estrictamente necesario para sobrevivir y a medida que aumenta la calidad de vida, se tiene la posibilidad de acceder a bienes de consumo que anteriormente simplemente no estaban al alcance de la población en general, y aparece el concepto denominado "Consumo de Masas."

Consumo, identificado con necesidades nuevas de la población como televisión frigorífico, teléfono automóviles que, en las últimas décadas desencadenan en una insatisfacción permanente, puesto que el consumidor depende de modelos y ritmos externos que escapan a su propia soberanía. Lo que hoy se consume de forma deseable, mañana deja de serlo, aunque el producto 
PROCESOS URBANOS - Revista de Divulgación Científica Vol. 1 Enero - Diciembre 2014 (63 - 70)

Tabla 1. Posesión de Bienes de Consumo por cada 100 hogares (España)

\begin{tabular}{|c|c|c|c|}
\hline & Año 1960 & Año 1964 & Año 1976 \\
\hline Televisión & 1 & 8 & 87 \\
\hline Frigorífico & 4 & 9 & 49 \\
\hline Automóvil & 4 & 8 & 44 \\
\hline Batidora & 4 & 15 & 49 \\
\hline Tocadiscos & 3 & 8 & 55 \\
\hline Teléfono & 12 & 25 & 49 \\
\hline Lavadora & 19 & 33 & \\
\hline
\end{tabular}

Fuente Editorial Trotta Serie Medio Ambiente 1997. Consumo y Medio Ambiente E. Kostka Fernández y Jesús Gutiérrez Brito.

del servicio sea el mismo y también el consumidor. Así se formaliza un consumismo cuyo rasgo característico es el incorporar al consumidor a una espiral sin fin donde constantemente ve relanzada su demanda.

A manera de ejemplo, encontramos los artículos tecnológicos utilizados en las actividades cotidianas. Día a día nos bombardean con nuevas prestaciones y servicios que implican de forma muy rápida la obsolescencia del producto, lo cual necesariamente conlleva a mayor consumo de bienes naturales $y$, por otro lado, mayor generación de residuos.

Adicionalmente, hay que tener en cuenta factores de orden social, lo cual en países como Colombia, la adquisición de ciertos bienes de consumo repercute en reconocimiento social, como puede ser la compra de un automóvil.

Ahora bien, es claro que se deben fortalecer las propuestas de un consumo sostenible, que protejan la gran riqueza en biodiversidad que posee nuestro país, pero también sería interesante enfocar estas tendencias para crear oportunidades de empleo y estructuración de iniciativas comunitarias que disminuyan la brecha social y se conviertan en factores de desarrollo para la comunidad, circunstancia que reviste la mayor importancia en nuestro país teniendo en cuenta la grave problemática social que lo afecta, entre otras razones, por falta de oportunidades laborales. A manera de ejemplo, encontramos cómo la protección a la biodiversidad se convierte en un motor de riqueza, para el país.

Importancia de la biodiversidad como base de la economía nacional: ${ }^{6}$

En producción agropecuaria desde 1994 contribuye con $10-14 \%$ del PIB nacional (Jarvis 2009) en producción pesquera y acuícola produce 166.000 Ton/año (50\% pesca industrial, $23 \%$ pesca artesanal y $27 \%$ productos de la acuicultura) (MADR 2008), en peces ornamentales durante el 2005: exportación de peces ornamentales US\$6.2 millones (Gómez y Ortega, 2007) en turismo representa $2.2 \%$ del PIB nacional con 380.000 empleos directos $(2.2 \%$ de empleos en el país) (Mincomercio) 2008) en ecoturismo en parques nacionales con 411.476 visitantes/ año se generan ( $\$ 2.3$ y $\$ 6.9$ mil millones de pesos y en plantas medicinales y aromáticas el consumo nacional pasó de 7 millones

${ }^{6}$ Presentación Ministerio de Ambiente, Vivienda y Desarrollo Territorial en el Curso de Capacitación Teórico -Práctico Sobre Técnicas de Manejo e Identificación de Especies Silvestres e Investigación Criminal Ambiental -DIJININTERPOL, octubre de 2010 Bogotá. 
de dólares para el 2002, a 23 millones de dólares en el 2007. genera 8.200 empleos. (Gómez y Ortega, 2007).

Por otra parte, también es importante enumerar los beneficios que la población obtiene de los ecosistemas, entre los cuales podemos destacar 1) aprovisionamiento - bienes producidos o proveídos por los ecosistemas (alimentos, fibras, agua dulce, madera, recursos genéticos minerales 2) regulación - beneficios obtenidos producto de la regulación de los procesos eco sistémicos (regulación del clima, control de plagas, regulación de inundaciones, polinización, dispersión de semillas, control de la erosión 3) culturales, beneficios no materiales de los ecosistemas (Espirituales recreacionales, estéticos y educativos 4) de soporte- servicios necesarios para la producción de otros servicios eco sistémicos (Formación de suelo y minerales, Formación de $\mathrm{O}_{2}$ atmosférico, reciclaje de nutrientes, producción primaria, provisión de hábitat, ciclo del agua, flujo de energía solar.

Dentro de este contexto, consideramos que para lograr la sostenibilidad ambiental deseada en nuestros entes territoriales, debemos iniciar un trabajo de fortalecimiento institucional que nos permita brindarles instrumentos concretos a los tomadores de decisiones, respecto al cuidado y protección de nuestros recursos naturales $y$, a su vez lograr el aprovechamiento comercial de los mismos, que sin lugar a dudas, cerraría la brecha social y redundaría en una mejora en la calidad de vida de nuestra población.

\section{- Uso sostenible}

A raíz del informe sobre nuestro futuro común y la cumbre de Rio de Janeiro de 1992, se comenzó a utilizar concepto de desarrollo sostenible a nivel mundial, concepto que dependiendo de quien lo utilice, merece diversas interpretaciones, sin embargo, a nuestro entender, desarrollo sostenible es aquel que satisface las necesidades del presente sin comprometer las de las futuras generaciones". Meta que se debe trabajar, implementando mediante estrategias productivas y educativas que permitan el rescate cultural, el desarrollo económico y social, dentro de un marco que busque la reorientación de prácticas sostenibles de los recursos vegetales, para que así la riqueza biológica se traduzca en riqueza social y económica.

Nuestro país cuenta con un extenso recurso filogenético que puede ser utilizado potencialmente a nivel industrial, medicinal y alimenticio, sin embargo, también su territorio se encuentra amenazado debido a la fragmentación ocasionada en su mayoría por los procesos de intervención antrópica, como es el aumento de los procesos de industrialización, la expansión de la zona urbana y el continuo desplazamiento de pobladores hacia las grandes ciudades entre otros.

La expansión de la frontera agrícola tiene como efecto directo la reducción de las áreas disponibles para la conservación de los recursos naturales renovables; este efecto cobra mayor relevancia en las áreas estratégicas para la conservación del recurso hídrico, como lo son las partes altas de la cordillera y los páramos. Los procesos mencionados traen, entre otras consecuencias, la disminución de las poblaciones de especies silvestres que cumplen un rol específico en la estructura y funcionamiento del ecosistema, lo que desestabiliza las interacciones a nivel de comunidades naturales de plantas, animales y otros organismos.

Dentro de las especies vegetales que se encuentran afectadas, existen muchas que presentan amplios potenciales de uso, lo cual incluye plantas alimenticias, medicinales, tintóreas, entre otras. Estas especies han sido tradicionalmente subvaloradas o, en otros casos, explotadas solo a nivel local, haciendo extracción directa del medio, lo cual puede incrementar el deterioro de las poblaciones silvestres, llevando gradualmente a la extinción local o general.

\section{- Conservación}

Es un hecho notorio y de común utilización, referirse a la gran riqueza en biodiversidad que posee nuestro país, pero también es innegable que esta riqueza paulatinamente 
viene disminuyendo. Entre los aspectos que podemos destacar, encontramos la extracción selectiva y no planificada de recursos naturales con propósitos distintos a la provisión de materias primas, el abastecimiento de las demandas domésticas de los habitantes y las actividades de uso y producción del suelo en proporciones que generan daños de difícil reversión.

También es importante resaltar que, el proceso de conservación y restauración de nuestros ecosistemas, se torna de vital importancia, teniendo en cuenta el auge que posee en la actualidad el proceso de minería en nuestro país, el cual presenta una situación caótica en áreas protegidas, tales como los parques nacionales, o en áreas con alguna forma de restricción ambiental.

\section{CONCLUSIONES}

- Las estrategias de comando y control utilizadas para afrontar la problemática ambiental en el sector productivo, no han mostrado los resultados esperados en el ámbito internacional, esta premisa utilizada para el caso de un país como Colombia, nos lleva a concluir que, a pesar de contar con múltiples instrumentos coercitivos en materia ambiental, como un nuevo procedimiento sancionador o diversas herramientas de contenido policivo, no podemos esperar que sean la solución. Necesariamente una política en la materia, debe crear herramientas de mercado, acuerdos de autogestión y fortalecer las relaciones entre lo público y privado.

- Indudablemente la técnica y el desarrollo científico aplicado a la industria ha llevado al hombre a elevar su calidad de vida; podemos ver cómo estas aplicaciones en ramas como la medicina han controlado las epidemias y plagas que asolaban a la humanidad en siglos anteriores; no obstante, estos adelantos en la mayoría de los casos, suponen la utilización irracional de los recursos naturales.
- Frente al desenfrenado auge consumista, en el ámbito internacional se viene presentando un fenómeno de sensibilidad ambiental, el cual promueve el respeto por el medio ambiente y la escogencia de procesos productivos limpios y que minimicen el impacto ambiental en la elaboración de bienes y servicios, es lo que comúnmente se ha denominado como un consumo ecológico, entendiéndolo como la preocupación por el efecto ambiental del producto consumido.

- No obstante, este consumo ecológico no necesariamente implica menos consumo, porque de todas maneras se sigue causando un impacto a los recursos naturales; lo que se debe trabajar y fortalecer en una política ambiental son los cambios de hábitos del consumidor actual, mediante estrategias de sensibilización que lleven implícito un mensaje de ética ambiental y contenido económico en su interior.

- Sería interesante enfocar las iniciativas de consumo sostenible hacia la utilización racional de nuestra riqueza en biodiversidad, con el fin de crear nuevas fuentes de empleo.

\section{REFERENCIAS}
Betancur Rodríguez, Andrés (2001) Instituciones de Derecho Ambiental Ed. La Ley, Madrid, pág. 171.

Santaella Héctor (2008) Lecturas sobre Derecho del Medio Ambiente. Editorial Universidad Externado de Colombia. 